\title{
Multivariable Process Identification for MPC: The Asymptotic Method and its Applications
}

\author{
Yucai Zhu \\ Tai-Ji Control \\ Hageheldlaan 62, NL-5641 GP Eindhoven \\ The Netherlands \\ Phone +31.40.2817192, fax +31.40.2813197, y.zhu@ tip.nl
}

\begin{abstract}
In this work we will introduce the asymptotic method (ASYM) of identification and provide two case studies. The ASYM was developed for multivariable process identification for model based control. The method calculates time domain parametric models using frequency domain criterion. Fundamental problems, such as test signal design for control, model order/structure selection, parameter estimation and model error quantification, are solved in a systematic manner. The method can supply not only input/output model and unmeasured disturbance model which are asymptotic maximum likelihood estimates, but also the upper bound matrix for the model errors that can be used for model validation and robustness analysis. To demonstrate the use of the method for model predictive control (MPC), the identification of a Shell benchmark process (a simulated distillation column) and an industrial application to a crude unit atmospheric tower will be presented.
\end{abstract}

\section{Introduction}

In the last decade, model predictive control (MPC) technology has gained its industrial position in refinery and petrochemical industries and is beginning to attract interest from other process industries. The most difficult and time consuming work during a typical industrial MPC project is modelling and identification (Richalet, 1993). Generally, identified black-box models are used for MPC controllers. There is a feeling in the field of process control, and especially model predictive control, that the development of identification is behind that of the control algorithms; see, e.g., Cott (1995a). The problem of process identification for MPC starts to attract attentions of both academic institutes and industry (Cott, 1995a, 1995b).

Although multivariable control concept is well accepted by MPC industry, the guideline for identification practice is still single-variable based thinking. The identification experiments are called step tests, which reflects the fact that each manipulating variable (MV) is stepped separately and some clear step responses are expected for modelling each transfer function. This approach works for stable processes, but the cost is very high. The product qualities may be disturbed by stepping the MVs. The test time is very long, which occupies much manpower and makes production planning difficult. The tests are done manually, which dictates extremely high commitment of the engineers and operators; it is not unusual that such tests are carried out around the clock. Worst of all, the models derived from this approach are not 
accurate for complex and highly disturbed processes. This in turn causes control performance problems during controller commissioning.

Some effort has been made to improve identification of HPI processes for MPC applications. One way is to use PRBS (pseudo random binary sequence) as test signals for single variable tests; see, e.g., Treiber et.al. (1992). This will reduce the disturbance to the product qualities. Another approach is to estimate multivariable FIR (finite impulse response) models using the data from a sequence of single variable tests; see Cutler and Hawkins (1988). This allows the operator intervention during the tests, which is helpful for reducing the disturbance to the production. These approaches are indeed a little bit more systematic and operation friendly than pure step test approach, but many trial-and-errors are still left to the user, and hence the costs of identification remain high. Fundamental problems of multivariable identification, such as optimal test design for control, model order/structure selection, model error quantification and model validation are not solved by these methods.

Wide spread applications of MPC technology call for more effective and efficient method of multivariable process identification.

In control community, system (process) identification has been one of the most active branch in the last three decades. SISO model identification was treated in the 70's, MIMO identification are studied in the 80 's, in the 90 's, control relevant identification attracts much attention. Also some new parameter estimation methods are emerging, such as subspace method, frequency domain method and estimation using orthonormal base functions.

An astonishing fact is that most of the identification results developed in the last 30 years are not used by industrial control engineers, although there is an urgent need for efficient and effective identification methods in process control industry. A reason for this failure of technology transfer is that most researchers follow a reductionism approach in their work. Too many people concentrate on parameter estimation and convergence analysis, a small piece of identification technology as a whole, while too few people study test design and model validation, the part that is close to model applications. Another reason is possibly due to the toolbox style of many identification software. Although toolbox approach is very flexible and user-friendly for researchers and students, this class of software is difficult to use by practical control engineers who do not have academic training in system identification.

In this paper we will introduce the so called asymptotic method (ASYM) of identification and show its use for MPC. The ASYM method was originally developed for linear robust control; see Zhu et. al. (1991) and Zhu and Backx (1993). The purpose of this paper is not to present new theory, or, to carry out analysis of existing method, rather, we will show how ASYM can be tailored for use in MPC and what improvement can be made by ASYM in comparison with current industrial identification practice.

In Section 2 the characteristics of refinery/petrochemical processes are discussed and the requirements of identification of these processes for MPC are outlined. In Section 3 the identification approach is introduced with MPC as the intended model application. In order to thoroughly examine the capability of the technology, two examples are presented. In Section 4 , a demonstration of the use of ASYM is given using a Shell benchmark process. In Section 5, the ASYM is used to identify a crude unit atmospheric tower for DMC control. Section 6 contains the conclusions and perspectives. 


\section{Identification Requirements for Use in MPC}

Before starting the presentation of the identification method, we will first discuss the requirements of identification for MPC applications. We will focus on processes in refinery and petrochemical industries where MPC application is most challenging and perhaps also most beneficial. This does not mean, however, that our discussion does not hold for other industries. This class of processes can be characterised as follows:

Continuous process. This is the only "good" character of the process class. The fact that these processes are operating in continuous mode makes it feasible to use linear time-invariant dynamic models for control.

Large scale and complex. A small MPC controller will have 3 to 5 MVs (manipulated variables or inputs) and 5 to $10 \mathrm{CVs}$ (controlled variables or outputs); a large size MPC will have 10 to $20 \mathrm{MVs}$ and 20 to $40 \mathrm{CVs}$. Some CVs, such as product qualities, are very slow (with dominant time constant range from 30 minutes to several hours), and other CVs are very fast, such as valve positions (with time constant in few minutes). Inverse response (nonminimum phase behaviour), oscillating behaviour and time delays exists. For a large controller, most often over $50 \%$ of the process transfer functions are practically zero and they need to be located.

Dominant slow dynamics. The time to steady state of a typical product quality model ranges from one hour to several hours. This dictates relative long time for identification test.

High level and slow disturbances. Typical source of unmeasured disturbances are feed composition variations and weather changes. These are slow and irregular variations. During an identification test, the level of disturbances is in average above $10 \%$ of that of $\mathrm{CV}$ variation (in power), but it can be as high as $40 \%$. Too large test signal amplitudes are not permitted because they will cause off-specification of product and/or nonlinearity.

Local nonlinearity. Although in general linear models are relevant for MPC for this class of processes for a given range of operation, some nonlinear behaviour may still show up. Examples are CVs that are very pure product qualities and valve positions close to their limits.

Based on these observations we will outline the special needs in identification for MPC and give comments on the existing methods.

Identification tests. A good identification test plays a dominant rule for a successful identification. Current practice of MPC industry is to use a series of single variable step tests for model identification. The tests are carried out manually. The advantage of this test method is that control engineer can watch many step responses during the tests and can learn about the process behaviour in an intuitive manner. The biggest problem of single variable step test is its high cost in time and in manpower. For example, to test a crude unit atmospheric tower will take two to three weeks around the clock with one control engineer on site. The second problem is that the data from single variable test may not contain good information about the multivariable character of the process and that step signals may not excite enough dynamic information of the process. These problems can be solved by using automatic multivariable test approach. An important issue of identification test is test signal design. Common optimal signal design methods proposed in literature try to minimise some measure of the parameter covariance matrix. This approach may not lead to useful result for control applications. For example, the optimal input that minimises the variances of finite impulse response parameters is white noise which we will show in Section 3 is not a good signal for MPC. For MPC application, a good design method is to choose the test signals that minimise the prediction 
error (often called simulation error in identification literature) under CV variation constraints. Ideally, if possible, the identification test should be done in closed-loop operation. One advantage of closed-loop test is obvious: the controller helps to keep CVs within their limits. What is less obvious is that a model identified from closed-loop operation gives better control performance (Hjalmarsson et al., 1996) provided that the identification method can handle closed-loop data properly.

Model structure, parametric or nonparametric. Several industrial identification software are based on finite impulse response (FIR) model. FIR models are called nonparametric models. This model class has inherent problems for use in identifying processes with slow dynamics. Model variance error is high due to its high order; yet bias error is often not negligible due to truncation. Therefore, parametric or compact models are preferred for identification of processes with slow dynamics. This is true also for MPC controllers that use non-parametric models. Of course, there are challenging problems in parameter estimation and order selection when using parametric models.

Criterion for model estimation. As mentioned before, slow and high level unmeasured disturbances are present. This means that when model accuracy is concerned, an estimation criterion that includes disturbance model will be better than a criterion without the disturbance model. Note that this is true even when disturbance model is not needed by the controller. Prediction error criterion and frequency domain maximum likelihood criterion belong to the first class; while output error criterion belongs to the second class. Moreover, prediction error criterion and maximum likelihood criterion will work properly for closed-loop data (model estimates are consistent meaning that the effect of the disturbance will decrease when test time increases); while output error criterion will deliver biased model when using closed-loop data. Several industrial identification software estimate FIR parameters using output criterion. This partly explains why industrial identification tests are mostly carried out in open-loop operation, although people realise the benefits of closed-loop identification.

Model validation. The goal of model validation is to test whether the model is good enough for its purpose and to provide advise for possible re-identification if the identified model is not valid for its intended use. Commonly used methods of model validation are simulation using estimation data or fresh data, whiteness test of model residuals and testing the independence between the residuals and past MVs. These methods only tell how well the model agrees with the test data. They can neither quantify the model quality with respect to the purpose of modelling, in our case, closed-loop control, nor can they give good advice for re-identification. Based on this observation, we think that the fundamental problem of model validation is basically unsolved in both literature and practice, especially for multivariable processes. Trial-and-error approach in this step makes industrial identification a very expensive practice. In linear model identification for control, a logical approach for model validation is to quantify model errors in the frequency domain and to find relationship between this kind of error description and model based control performance. Model error estimation has attracted much interests in the last decade, this was motivated mainly by linear robust control theory.

Software user interface. In an engineering company, most MPC users are not identification experts. Often process identification is the most difficult and time consuming part of an MPC project. A desired identification technology should be able to provide simple guidelines for good identification tests and when test data are collected, to carry out various calculations systematically or even automatically without the necessity of user intervention. Most existing identification software developed by identification experts follows toolbox approach so that 
the user can try various methods/techniques on each steps. This is certainly very convenient for academic researchers and students, but it is rather user-unfriendly for industrial MPC users. The user-friendliness of an identification method for industrial users depends heavily on its ability to solve various fundamental problems in process identification.

\section{Asymptotic Method of Identification}

The asymptotic method (ASYM) of identification was originally developed for the purpose linear robust control; see Zhu et. al. (1991) and Zhu and Backx (1993). We will show here that with little modification, the method can be made suitable for MPC applications. Based on an asymptotic theory, we will give practical procedure for identification test design and model validation which are week links between theory and applications.

The name asymptotic method reflects the fact that the approach is based on the asymptotic theory of identification developed by Ljung and Yuan; see Ljung (1985), and Ljung and Yuan, (1985) for single variable version and Zhu (1989) for multivariable extension. Given a multivariable process with $m$ manipulated variables (inputs) and $p$ controlled variables (outputs). Denote the data sequence that is collected from an identification test as

$$
Z^{N}:=\{u(1), y(1), u(2), y(2), \ldots \ldots, u(N), y(N)\}
$$

where $u(t)$ is $m$-dimensional input vector, $y(t)$ is $p$-dimensional output vector and $N$ is the number of samples.

We assume that the data is generated by a linear discrete-time process as

$$
y(t)=G^{o}\left(z^{-1}\right) u(t)+H^{o}\left(z^{-1}\right) e(t)
$$

where $z^{-1}$ is the unit time delay operator. Here $H^{\mathrm{o}}\left(z^{-1}\right) e(t)$ represents the unmeasured disturbances acting at the outputs, and $e(t)$ is a $p$-dimensional white noise vector.

Remark: The assumption (3.2) may seem too restrictive to represent a complex industrial plant such as a crude unit. The success story of the MPC industry has shown that for most of the continuous processes, linear time-invariant models are good enough for the purpose of MPC control. Our experiences have shown that for important controlled variables, such as product qualities and column temperatures, linear models are adequate for MPC applications. Very high purity distillation columns may be exceptional. Valve positions are often used as constraint controlled variables and they often show nonlinearity when they are close to high/low limits. It is, however, not difficult to get around this problem. One way is to do some nonlinear compensation and another way can be simply de-tune the control for the valves (the model gain is larger when a valve is almost full open or closed).

The model to be identified is in the same structure as in (3.2):

$$
y(t)=G\left(z^{-1}\right) u(t)+H\left(z^{-1}\right) e(t)
$$

The process model $G\left(z^{-1}\right)$ and noise filter $H\left(z^{-1}\right)$ will be parametrized in matrix fraction description (MFD) with diagonal denominator matrices; see Zhu and Backx (1993) for details. The model will be calculated by minimizing the prediction error cost function; see Ljung (1985).

The frequency response of the process and of the model are denoted as

$$
T^{o}\left(e^{i \omega}\right):=\operatorname{col}\left[G^{o}\left(e^{i \omega}\right), H^{o}\left(e^{i \omega}\right)\right]
$$




$$
\hat{T}^{n}\left(e^{i \omega}\right):=\operatorname{col}\left[\hat{G}^{n}\left(e^{i \omega}\right), \hat{H}^{n}\left(e^{i \omega}\right)\right]
$$

where $n$ is the degree of the polynomials of the model, col(.) denotes the column operator.

\section{The Asymptotic Properties of Prediction Error Method}

Assume that

$$
\text { - } n \rightarrow \infty \text { and } n^{2} / N \rightarrow 0 \text { as } N \rightarrow \infty
$$

- Test signals have continuous none zero spectra until the Nyquist frequency.

Then (Ljung, 1986 and Zhu, 1989)

$-\quad \hat{T}^{n}\left(e^{i \omega}\right) \rightarrow T^{o}\left(e^{i \omega}\right)$ as $N \rightarrow \infty$

- The errors of $\hat{T}^{n}\left(e^{i \omega}\right)$ follows a Gaussian distribution, with covariance given as

$$
\operatorname{cov}\left[\hat{T}^{n}\left(e^{i w}\right) \approx \frac{n}{N} \Phi^{-T}(\omega) \otimes \Phi_{v}(\omega)\right.
$$

where $\Phi(\omega)$ is the spectrum matrix of inputs and prediction error residual $\operatorname{col}\left[u^{T}(t), \xi^{T}(t)\right]$, $\Phi_{v}(\omega)$ is spectrum matrix of unmeasured disturbances, $\otimes$ denotes the Kronecker product and $-T$ denotes inverse and then transpose.

Note that the theory holds for the general case of closed-loop test. For open-loop test, the following simplified results can be obtained for process model

$$
\operatorname{cov}\left[\operatorname{col}\left[\hat{G}^{n}\left(e^{i w}\right)\right] \approx \frac{n}{N} \Phi_{u}^{-T}(\omega) \otimes \Phi_{v}(\omega)\right.
$$

where $\Phi_{u}(\omega)$ is the spectrum matrix of inputs.

In the following, we will outline the ASYM method which makes extensive use of the asymptotic theory. In order to minimise the presentation complexity, we will outline the method for open-loop experiment and also assume that the test inputs are mutually independent.

\section{The Procedure of the Asymptotic Method}

\section{1) Identification test}

The following are the important features of the ASYM test:

- Duration of one test is about $10 \sim 15$ times the longest response time.

- Operator manual control is encouraged.

- The optimal test signal is designed to minimise the sum of the squares of the simulation error (often called prediction error in MPC term). The spectra of the test signals are based on the following formula which can be derived from (3.6) (see Ljung and Yuan, 1985 and Zhu and Backx, 1993):

$$
\Phi_{u_{j}}^{o p t}(\omega)=\mu_{j} \sqrt{\Phi_{u_{j}}^{s i m}(\omega) \sum_{i=1}^{p} \Phi_{v_{i}}(\omega)}
$$


where $\Phi_{u_{j}}^{\operatorname{sim}}(\omega)$ is the spectrum of the $j$-th input movement in a controlled system, $\mu_{j}$ is a constant which is adjusted to meet the amplitude constraint of the signal.

In practice, (3.7) is used in combination with upper error bound (3.12) to give general guidelines for the test design of various processes. The spectra of the test signals can be realised by PRBS (pseudo-random-binary-sequence) signals or filtered white noises. Take independent PRBS signals as an example. The character of a PRBS signal can be determined by its average switch time and its amplitude. The amplitudes of PRBS signals are determined by a priori knowledge of the process or by some short step test. Extensive study that applies (3.7) and (3.12) to many industrial process models show that the optimal average switch time of PRBS signals can be related to the longest process response time. It turns out that the optimal average switch time is about $1 / 3$ of the longest process response time. The longest process response time for a given process is often known from project experience, or, if not, it can be determined from some pre-test.

Comments on ASYM test design. The test time is set in order to fulfil approximately the conditions required for the asymptotic theory. In MPC control, model is used to simulate (predict) the process online and control actions are calculated using the predictions. Designing the test signals by minimising the simulation errors is certainly a good method for MPC applications. Theoretically optimal criterion for MPC control is difficult to obtain because of the multiple objective approach used by MPC control technology. In order to use the design formula, the user only needs to know the dominant time constant of the underlying process which is often available or, easily obtainable. This means that the user himself does not need to do iterations between identification and control in order to use the design method. Note that the design is done for the slow CVs because normally the model quality for fast CVs are better. The resulting test signals are typically much slower than white noise signals, but they are much faster than step signals.

Before model identification, some data pre-treatment is carried out. This includes removing the spikes, data slicing, hi-pass filtering (de-trending), known delay correction and signal scaling. This part of the work is well known; see Ljung (1987).

\section{2) Estimate a high order ARX (equation error) model}

$$
\hat{A}^{n}\left(z^{-1}\right) y(t)=\hat{B}^{n}\left(z^{-1}\right) u(t)+\hat{e}(t)
$$

where $\hat{A}^{n}\left(z^{-1}\right)$ is a diagonal polynomial matrix and $\hat{B}^{n}\left(z^{-1}\right)$ is full polynomial matrix, both with degree $n$ polynomials. Denote $\hat{G}^{n}\left(z^{-1}\right)$ as the high order ARX model of the process, and $\hat{H}^{n}\left(z^{-1}\right)$ as the high order model of the disturbance.

\section{3) Perform frequency weighted model reduction (ML estimate)}

The high order model in (3.8) is practically unbiased, provided that the process behaves linear around the working point. The variance of this model is high due to its high order. Here we intend to reduce the variance by perform a model reduction on the high order model. If we view the frequency response of the high order estimates as the noisy observations of the true transfer function, we can then apply the maximum likelihood principle. Using the asymptotic result of (3.4) and (3.6), we can show that the asymptotic negative log-likelihood function for the reduced process model is given by (Wahlberg, 1989, Zhu and Backx, 1993) 


$$
\mathrm{V}=\sum_{i=1}^{p} \sum_{j=1}^{m} \int_{\omega_{1}}^{\omega_{2}}\left|\left\{\left|\hat{G}_{i j}^{n}(\omega)-\hat{G}_{i j}(\omega)\right|^{2} \Phi_{u_{j}}(\omega) \Phi_{v_{i}}^{-1}(\omega)\right\}\right| \mathrm{d} \omega
$$

The reduced model $\hat{G}\left(z^{-1}\right)$ is thus calculated by minimizing (3.9) for a fixed order. The same can be done for the disturbance model $\hat{H}^{n}\left(z^{-1}\right)=1 / \hat{A}^{n}\left(z^{-1}\right)$. The reduced model is converted to diagonal ARMAX form:

$$
\hat{A}\left(z^{-1}\right) y(t)=\hat{B}\left(z^{-1}\right) u(t)+\hat{C}\left(z^{-1}\right) \hat{e}(t)
$$

where $\hat{A}\left(z^{-1}\right)$ and $\hat{C}\left(z^{-1}\right)$ are diagonal polynomial matrices.

\section{4) Use asymptotic criterion (ASYC) for order selection}

The best order of the reduced model is determined using a frequency domain criterion ASYC which is related naturally to the noise-to-signal ratios and to the test time; see Zhu (1994) for the motivation and evaluation. The basic idea of this criterion is to equalise the bias error and variance error of each transfer function in the frequency range that is important for control. Let $\left[\omega_{1}, \omega_{2}\right]$ defines the frequency band that is important for the MPC application, the asymptotic criterion (ASYC) is given by:

$$
\operatorname{ASYC}:=\sum_{i=1}^{p} \sum_{j=1}^{m} \int_{\omega_{1}}^{\omega_{2}}\left|\left[\left|\hat{G}_{i j}^{n}(\omega)-\hat{G}_{i j}(\omega)\right|^{2}-\frac{n}{N} \Phi_{u_{j}}^{-1}(\omega) \Phi_{v_{i}}(\omega)\right]\right| \mathrm{d} \omega
$$

\section{5) Model validation using error bound matrix}

According to the result (3.4) and (3.6), a $3-\delta$ bound can be derived for the high order model as follows:

$$
\left|G_{i j}^{o}\left(e^{i \omega}\right)-\hat{G}_{i j}^{n}\left(e^{i \omega}\right)\right| \leq 3 \sqrt{\frac{n}{N} \Phi_{u_{j}}^{-1}(\omega) \Phi_{v_{i}}(\omega)} \quad \text { w.p. } 99.9 \%
$$

We will also use this bound for the reduced model because the model reduction will in general improve model quality.

If the controller is linear and time-invariant, the upper bound matrix (3.12) can readily be used in robust stability and performance analysis, which is well known in linear robust control literature; see, for example, Kouvaritakis and Latchman (1985). For model predictive control where controller is monlinear and time-variant, no theory is available yet for the analysis of robust stability and performance (even the proof of nominal stability becomes a very challenging problem). In the following we will give two engineering solutions to the model validation problem based on the derived error bounds.

Validation method one - borrowing linear robust control theory. Design a linear controller that includes all the MVs but only important CVs so that the number of MVs is greater or equal to that of the CVs. Design a linear controller so that the closed loop response time is approximately the same as that for MPC controller. Analyse the robust stability and performance using the corresponding upper bound matrix. If the linear controller passes the test, the model will pass the validation. If the controller does not pass the test, the result of the analysis will indicate which frequency range cause the problem and this in turn will give information for test redesign. Note that the upper bound (3.12) is a function of two design variables: number of samples $N$ (test time) and MV spectrum. This method is suitable when 
linear robust control software is available and the user understands how to carry out robust control design or the control design is automated.

Validation method two - grading the models. This is done by comparing the relative size of the bound with the model over the low and middle frequencies. More specifically, identified transfer functions are graded in A (very good, bound $<=30 \%$ model), B (good, bound $<=60 \%$ model), $\mathrm{C}$ (marginal, bound $<=90 \%$ model), and D (poor, or, no model exists, bound $>90 \%$ model). Based on extensive simulations and project experience, usually A grade and $\mathrm{B}$ grade models can be used in the controller, provided that the process is not very illconditioned for important CVs. C grade and D grade models are not relevant for MPC control and they are treated as follows:

1) Zero them when there are no transfer between the MV/CV pairs. This can be determined by using the process knowledge and cross checking.

2) If a transfer function is expected and needed in the control, redesign a test in order to improve the accuracy of these models.

Using upper bound formula (3.12) we can easily give some guidelines for improving the test design:

- double the amplitudes of test signals or quadruple the test time will half the error over all frequencies;

- double the average switch time of PRBS signals will half the model error at low frequencies and double the error at high frequencies.

This validation method is more suitable for users who are not familiar with robust control design methods.

At this moment, a critical reader may ask how many iterations of test-validation-redesign are necessary in order to arrive at a relevant model for MPC control, because he is concerned with the high cost of test for slow processes such as distillation columns. In practice, for a slow process, we recommend the user to carry out an ASYM identification in the middle of a test and adjust the second half of the test according to the model validation results. So the answer to the question is (practically): one iteration.

Comments on ASYM model validation. Although we have not yet find a complete theoretical solution for model validation, we can answer, to a great extend, the real model validation question: is the identification model good enough for the MPC control? Yes, if the linear controller passes robustness test (in method one), or, all required transfer functions have grades A or B (in method two). No, if the linear controller does not pass robustness test, or, some required transfer functions have grades $\mathrm{C}$ or $\mathrm{D}$. If the model is invalidated, test redesign can be given. We admit that our yes and no here are with some fuzziness. This is most common when theory meets reality and we find that most engineers accept this way of practice.

Comments on closed-loop identification. If identification test is carried out in closed-loop operation, the ASYM can be modified as follows. Use formula (3.7) for the design of spectra of test signals and apply them on setpoints of CVs. Independent PRBS signals can be used as test signals. The number of applied test signals should be greater than or equal to the number of MVs because otherwise the process is not completely identifiable. If the process is partly in closed-loop, for example, only several CVs are under PID control, then the MVs that are not 
used by the controllers need to be excited as well. For closed-loop data, ASYM formulae (3.8) $\sim$ (3.12) should be modified using (3.5) instead of (3.6).

Because ASYM provides systematic solutions to all the four identification problems, it can be made very user friendly for non expert users. Recently, ASYM has been implemented in an automatic identification software Tai-Ji ID; see Zhu and Ge (1997).

\section{$4 \quad$ Identification of a Shell Benchmark Process}

The Shell benchmark process is a model of a two-input two-output distillation column. The model may be too simple to represent any real process. However, we choose this simulation example for the following purposes: 1) To show the ASYM method in a know process in order to verify the theory used by ASYM, such as input design, upper bound and order selection; 2) to compare the model accuracy with other methods for the same example; 3 ) to show how to locate a zero transfer function. Next section contains a real industrial application of ASYM.

The inputs are overhead vapour flow $(D)$ and reboiler duty $(Q)$; the outputs are column pressure $(P)$ and product impurity $(X)$. The model of the process is given as follows (Cott, 1995b):

$$
\begin{aligned}
P^{d}(t) & =\frac{-0.6096+0.4022 q^{-1}}{1-1.5298 q^{-1}+0.5740 q^{-2}} D^{d}(t)+\frac{-0.1055-0.0918 q^{-1}}{1-1.5298 q^{-1}+0.5740 q^{-2}} Q^{d}(t) \\
& +\frac{N_{s}}{1-1.5945 q^{-1}+0.5945 q^{-2}} e_{p}(t)
\end{aligned}
$$

where $P^{d}, D^{d}$ and $P^{d}$ are deviation variables for pressure, overhead vapour flow and reboiler duty respectively, and $e_{p}(t)$ is the white noise which generates the disturbance on the pressure. The parameter $N_{s}$ is used to set the noise level used in simulation. For this study three values are used: $0.2,0.5$ and 1 and they are called 20\%,50\% and 100\% noise level simulations. The standard deviation of $\left\{e_{p}(t)\right\}$ is 1.231 .

The model of the impurity $(X)$ is slightly nonlinear. It is given as follows:

$$
\begin{aligned}
X(t) & =0.0765 \frac{500000}{Q(t-7)}+0.9235 X(t-1) \\
& +\frac{N_{s}}{1-1.6595 q^{-1}+0.0 .6595 q^{-2}} e_{x}(t)
\end{aligned}
$$

The standard deviation of $\left\{e_{x}(t)\right\}$ is 0.677 . Note that the overhead flow does not affect the impurity.

\section{1) Identification Tests}

We decide to use PRBS test signals due to its simplicity and design rules outlined in the previous section is used. First the following three open-loop tests are designed and simulated: 


$\begin{array}{llll} & \mathbf{2 0 \%} \text { noise } & \mathbf{5 0 \%} \text { noise } & \mathbf{1 0 0 \%} \text { noise } \\ N_{s} & 0.2 & 0.5 & 1.0 \\ D \text { amplitude } & 10 & 10 & 10 \\ \text { Average switch time } & 10 & 10 & 10 \\ Q \text { amplitude } & 200 & 200 & 200 \\ \text { Average switch time } & 20 & 20 & 20 \\ \text { Total length } & 1000 & 1000 & 1000\end{array}$

Comparing these tests with the five groups summarised in Cott (1995b), our test time is the shortest, the $D$ amplitude is in the middle of the five groups, the average PRBS switching time of $D$ is in the low side, the $Q$ amplitude is smallest, and its average PRBS switching time is in the low side. This makes our tests second least disturbing tests among different groups (Shell design is least disturbing).

The inputs and outputs are differenced for removing the nonstationary component of the output disturbances.

2) Model identification. In the identification sessions, the process was treated as a blackbox and no a priori knowledge of model structure is used except the assumption of linearity. The MISO model orders are determined by using the ASYC and no explicit time delays are estimated. The following tables summarise the model order selection during the identification. For each MISO model, the lowest order is the first try, and then higher order is used. The orders with minimum ASYCs are selected and they are underlined in the tables.

\begin{tabular}{c|c|c|c|c|c|c|c}
$20 \%$ \\
Order & 2 & 3 & 4 & 5 & 6 & 7 & 8 \\
\hline $\begin{array}{c}\text { ASYC of } \\
\text { P model }\end{array}$ & 20.8382 & 0.1401 & $\underline{0.0614}$ & & & & \\
\hline $\begin{array}{c}\text { ASYC of } \\
\text { X model }\end{array}$ & & & & 10.9584 & 5.8119 & $\underline{4.6782}$ & 5.3347
\end{tabular}

$50 \%$ noise case

\begin{tabular}{c|c|c|c|c|c} 
Order & 3 & 4 & 5 & 6 & 7 \\
\hline $\begin{array}{c}\text { ASYC of } \\
\text { P model }\end{array}$ & 0.2412 & $\underline{0.1708}$ & & & \\
\hline $\begin{array}{c}\text { ASYC of } \\
\text { X model }\end{array}$ & & 169.153 & 28.302 & 22.898 & $\underline{20.514}$
\end{tabular}

$100 \%$ noise case

\begin{tabular}{c|c|c|c|c|c} 
Order & 3 & 4 & 5 & 6 & 7 \\
\hline $\begin{array}{c}\text { ASYC of } \\
\text { P model }\end{array}$ & $\underline{0.6709}$ & 0.6723 & & & \\
\hline $\begin{array}{c}\text { ASYC of } \\
\text { X model }\end{array}$ & 77.4509 & 63.9533 & 30.8629 & $\underline{29.427}$ & 35.0011
\end{tabular}

The step responses of the process and of the three models are plotted in Figure 4.1. 

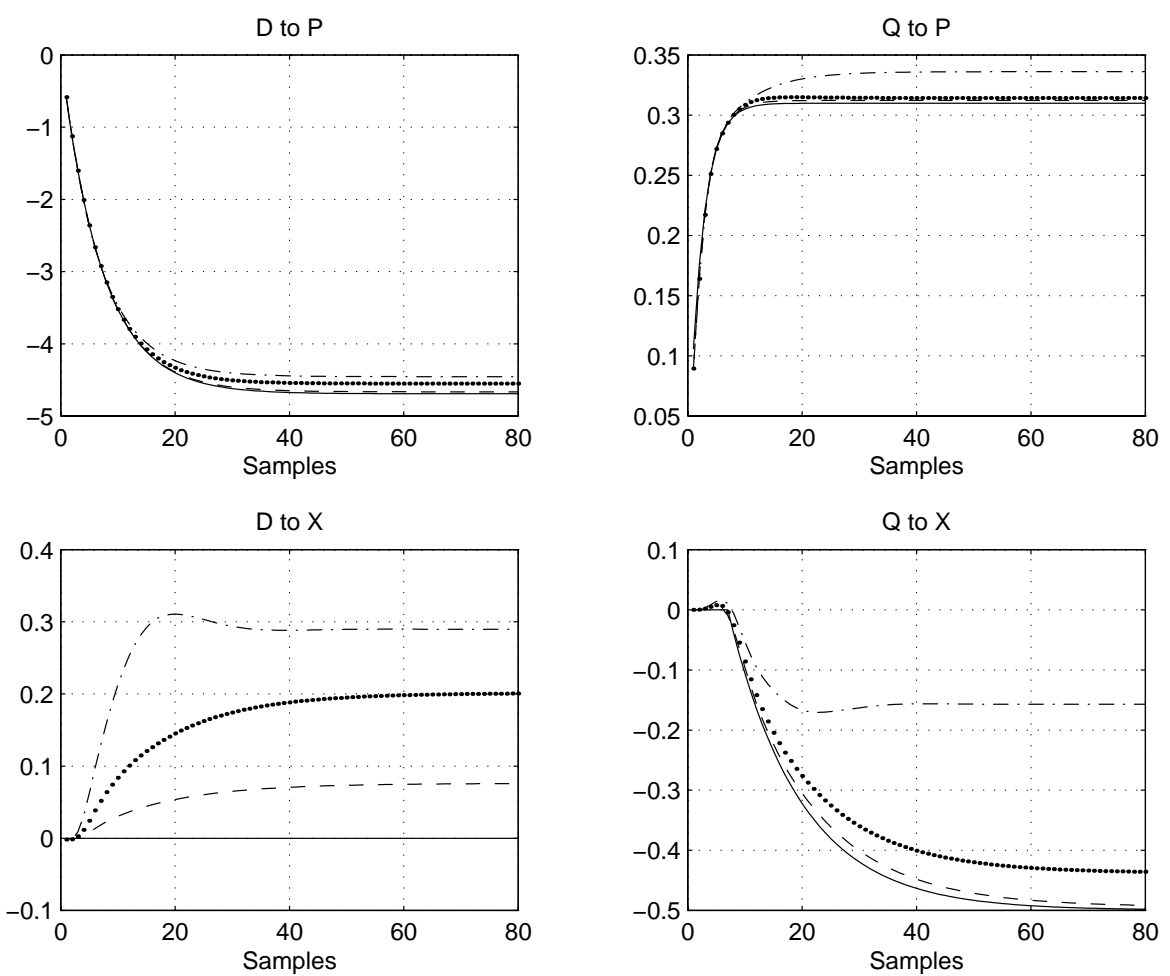

Figure 4.1 Step responses of the process and of the three models.

"Solid" := process, "dashed" := 20\% noise model, "dotted" := 50\% noise model "dash dot" := 100\% noise model.

We find that all the step responses are accurate except the one from duty $Q$ to impurity $X$ in the $100 \%$ noise case. We also note that the identified models in all three cases have non zero step responses from overhead flow $D$ to impurity $X$ while the true one is zero.

In Figure 4.2 and 4.3 the frequency responses of identified models and estimated upper error bounds for $50 \%$ and $100 \%$ noise cases are plotted.

SN50DIS: Bode plots (mag) and error bounds
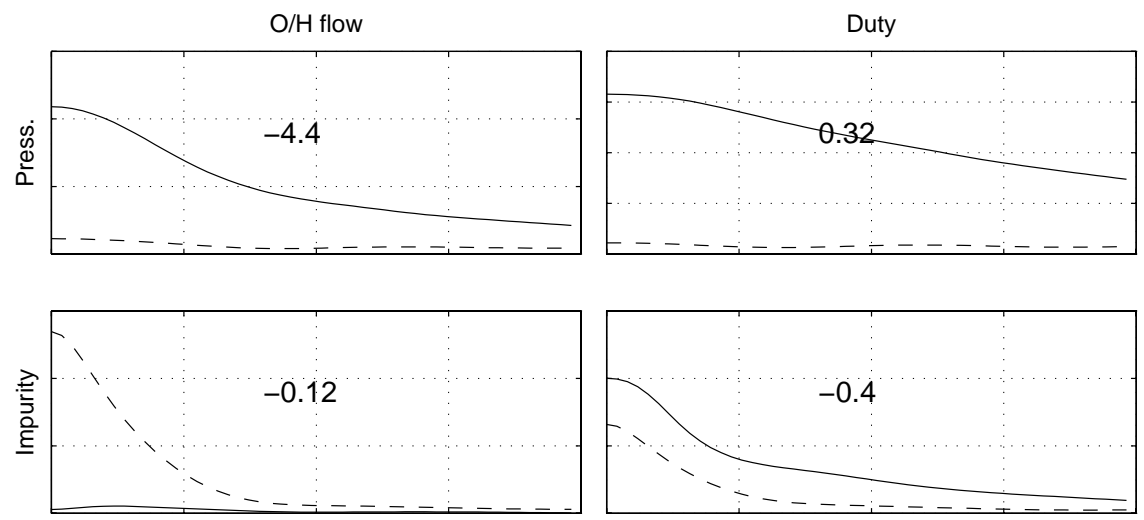

Norm. freq [rad/min], total 0.785398 .

Figure 4.2 Frequency responses of the high order model (solid) and upper bounds (dashed) of the $50 \%$ noise case 


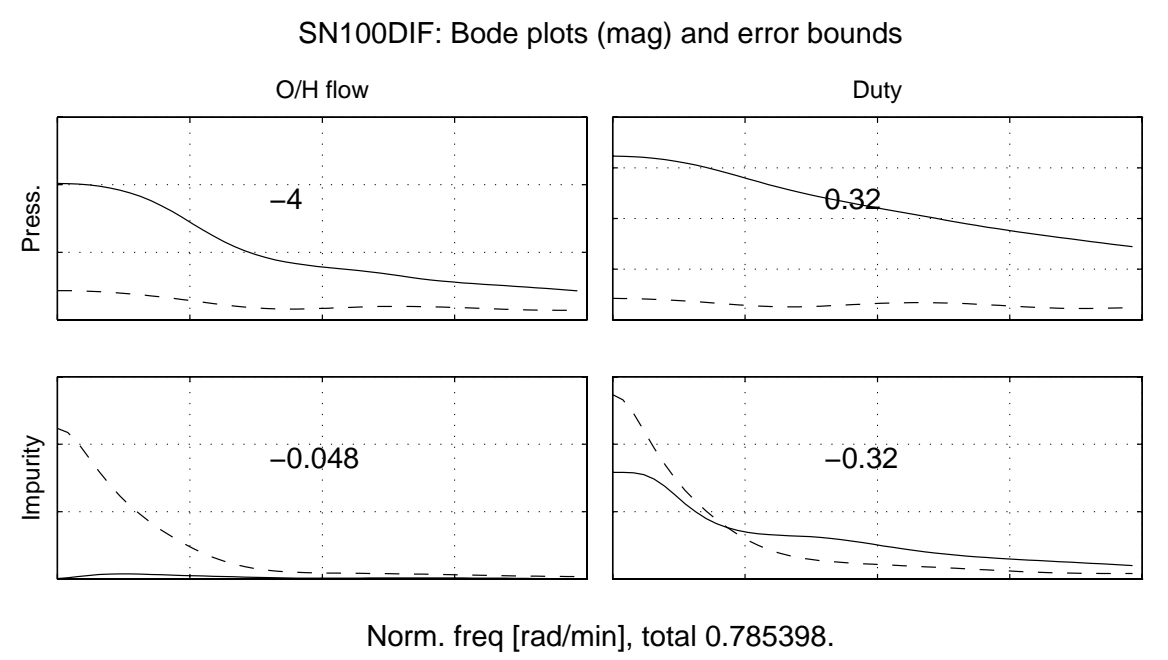

Figure 4.3 Frequency responses of the high order model (solid) and upper bounds (dashed) of the $100 \%$ noise case

The upper bounds show that pressure models are very accurate in all noise level. The upper bounds are many times greater than the model for the transfer function from $D$ to $X$. This can either be caused by too small amplitude of $D$ or there is no model here. It is easy to confirm that there is practically no model, because, by cross checking the transfer function from $D$ to $P$ we find a very good model.

Summarising, for the $20 \%$ and $50 \%$ noise cases, all models are A (very good) or B (good), there is no model between $D$ and $P$. Therefore, these models can be used for control. The only problem is the transfer function from $Q$ to $X$ in the $100 \%$ noise case where the model gain is too low. However, this poor model is clearly indicated by the upper bound in model validation which tells us the model error in the low frequencies can be large; see Figure 4.3.

What can we do to increase the model accuracy for this poor model? Our approach is to redesign a test. There are three possibilities in modifying the previous test: 1) change the spectrum of $Q ; 2$ ) increase the test time; and 3) increase the $Q$ amplitude. Here we will increase both the $Q$ amplitude and the test time by $50 \%$ and we will perform the following open-loop test:

$\begin{array}{ll}N_{s} & 1.0 \\ D \text { amplitude } & 10 \\ \text { Average switch time } & 10 \\ Q \text { amplitude } & 300 \\ \text { Average switch time } & 20 \\ \text { Total length } & 1500\end{array}$

The identified model is compared with the true model in Figure 4.4. We see that the transfer function from $Q$ to $X$ is improved considerably and more significant is that we can tell this in model validation; from Figure 4.5 we see a B (good) model between $Q$ and $X$. 

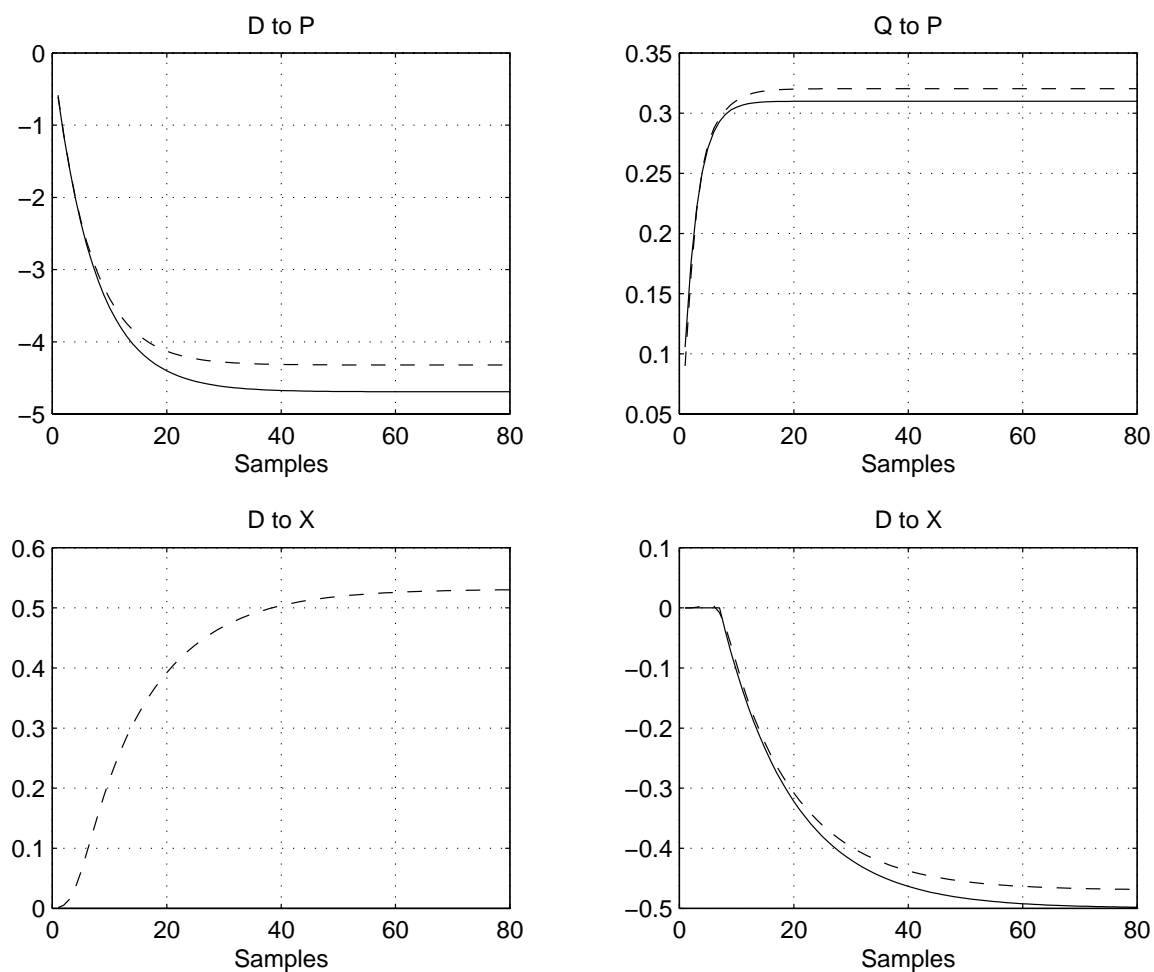

Figure 4.4 Step responses of the process and of the new model. "Solid" := process, "dashed" := 100\% noise model, new test,

SN100DI2: Bode plots (mag) and error bounds
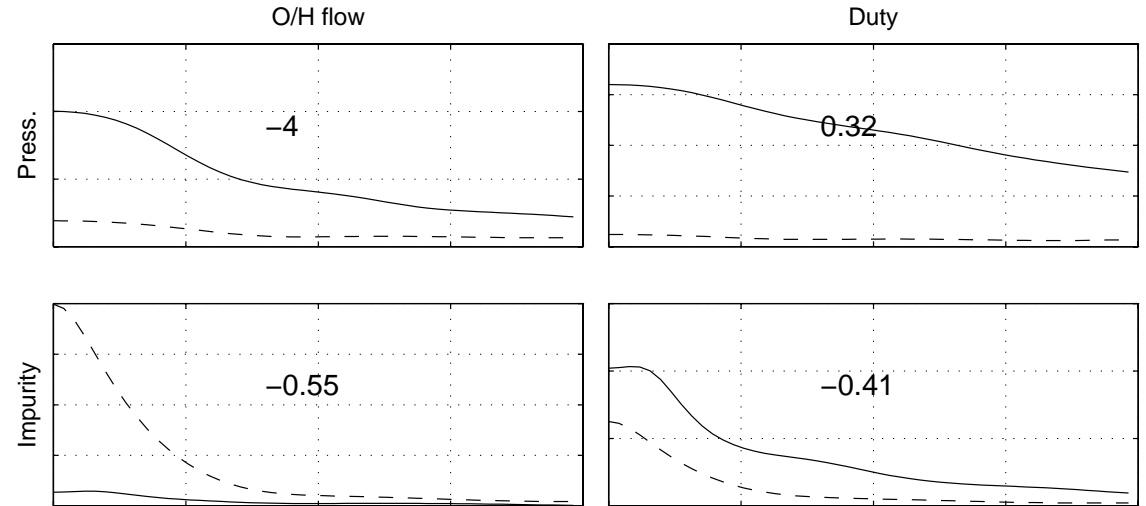

Norm. freq [rad/min], total 0.785398

Figure 4.5 Frequency responses of the high order model (solid) and upper bounds (dashed) of the $100 \%$ noise case, new test

The reader may compare the model quality of the ASYM models identified here with the models from five other methods for the same process; see Cott (1995b). 


\section{Identification of a Crude Unit for DMC Control ${ }^{1)}$}

In the last few years, the ASYM method has been applied successfully to many refinery and petrochemical units during various MPC projects. In this section we will show the use of the ASYM during a crude unit DMC project.

The crude unit consists of three distillation columns in series: an atmospheric tower, a splitter and a stabilizer column. Two DMC controllers have been installed for the crude unit, one for atmospheric column and one for splitter/stabilizer. In this paper we will only discuss the first controller. Figure 5.1 shows a simplified process flow diagram of the atmospheric tower. The column performs the initial distillation of the crude oil into various boiling range fractions. The column has four side draws each with its own side draw stripper:

- Kerosene (side draw 1),

- In-line blended automotive diesel (AD) (combined side draws 1,2,3,4 and an intermediate gas oil (IGO) from the vacuum unit)

- Heavy gasoil (side draw 4).

The column has whole straight run naphtha (WSR) as top product and a bottom residue is the feedstock for the vacuum unit. Moreover the column has a top reflux flow and three pump around flows of which the TPA and BPA exchange heat with stabilizer and splitter columns respectively.

The operating economics of a crude unit generally dictate that the unit should run as close as possible to the product specifications. More specifically the DMC control objectives are:

\section{Minimum naphtha mode:}

- Minimise naphtha flow.

- Maximise kero flow at minimum flashpoint and maximum 90\% point specifications.

\section{Off minimum naphtha mode:}

- Maximise naphtha flow at maximum $90 \%$ point specification of the HSR-naphtha.

- Kero $90 \%$ point at maximum specification. Flashpoint is no longer a control target.

\section{Always:}

- Maximise automotive diesel yield up to process limits and cloudpoint specifications at the expense of heavy gasoil (HGO).

- Maximise HGO yield up to process limits and color specification at the expense of residue yield.

- Maximise heater outlet temperature.

- Maximise crude feed flow up to process limits.

- Minimise column pressure.

Maximising heat recovery in crude preheat exchangers minimises heat loss in air-fin coolers. This in turn requires:

- Maximise TPA flow to maximise preheat up to stabilizer energy needs.

\footnotetext{
1) The work of the DMC project was carried out when the author was with AspenTech Europe BV. The author would like to thank the management and colleagues of the company for the encouragement and help during the work.
} 
- Maximise BPA flow to maximise preheat up to splitter needs.

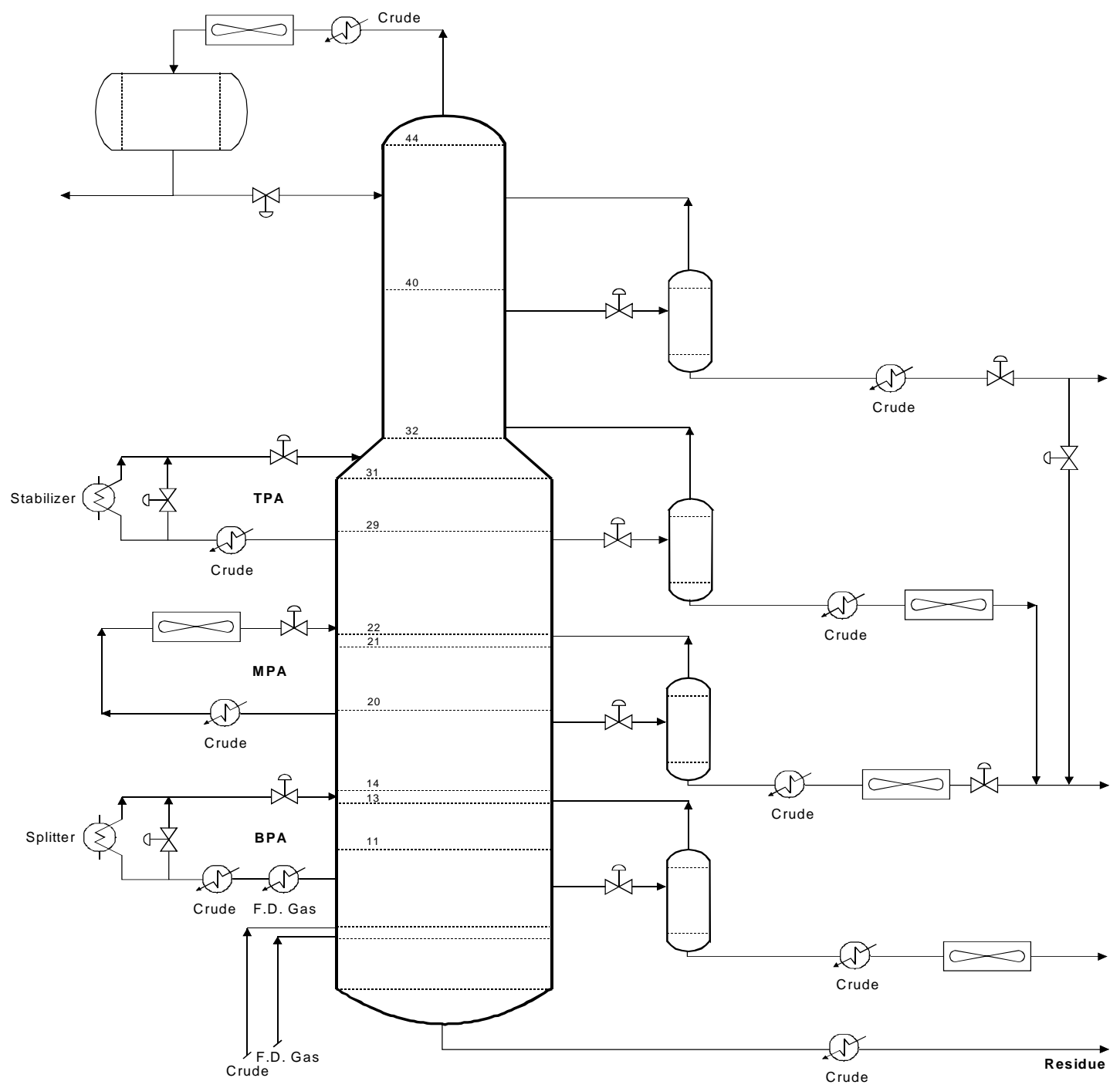

Figure 5.1 Simplified flow diagram of crude atmospheric distillation column

In order to achieve above mentioned control objectives, the following MVs, disturbances and outputs have been selected:

\section{Manipulating variables (MVs), or, inputs:}

1. 40FRC62S:

2. 40FRC63S:

3. 40FRC96S:

4. 40TRC50S/60S:

5. 40FC184S:

6. 40FC185S:

7. 40FC186S:

8. 40FC187S:

9. 40FRC18S:

10. 40FRC14S:
Crude feed flow rate

Crude feed flow rate

Crude feed flow rate

Combined coil outlet temperature

1st side draw flow rate

2nd side draw flow rate

3rd side draw flow rate

4th side draw flow rate

1st side stripper steam flow rate

2nd side stripper steam flow rate 
11. 40FRC15S:

12. 40FRC38S:

13. 40FRC10S:

14. 40FRC90S:

15. 40FRC11S:

16. 40FRC35S:

17. 40FRC79S:

18. 40FC170S:

19. 2FRC22S: 3rd side stripper steam flow rate

Top reflux flow rate

Light reflux flow rate

Medium reflux flow rate

Heavy reflux flow rate

Splitter feed flow rate

1st side flow rate to automotive diesel

4th side flow rate to automotive diesel

IGO flow rate to automotive diesel

Disturbance variables (DVs), or feedforward variables:
1. 40PR8:
Steam pressure
2. 40SRC4:
Compressor feed
3. 40FR98:
Flash drum off-gas flow

Controlled variables (CVs), or, outputs:

1. 2FRC22X:

2. 2LRC4X:

3. 40FRC79X:

4. 40FRC79S:

5. 40FRC9X:

6. 40FC170X:

7. 40FRC1S:

8. 40FRC1X:

9. 40LRC9X:

10. 40LRC7X:

11. 40LRC8X:

12. 40LRC6X:

13. 40LRC5X:

14. 40FRC7S:

15. 40FC184:

16. 40FC185:

17. 40FC186:

18. 40FC187:

19. 40PCT4/40TRC4:

20. 40AR4:

21. 40KERO90/40TBPSC 1

22. 40AR6:

23. 40GASOIL:

24. 40AR46:

25. 40LRC16:

26. 40TRC11X:

27. 40TRC13X:

28. 40FRC62X:

29. 40FRC63:

30. 40FRC96:

31. 40CRDHVO:
IGO flow valve position

IGO drum level control valve position 40FRC79 valve position

Flow rate to MEROX

40FRC9 valve position

40FC170 valve position

1st side draw to tank flow rate

40FRC1 valve position

1 st side stripper level control output

2nd side stripper level control output

3rd side stripper level control output

4th side stripper level control output

Bottom level control output

Residue flow rate

40FC184 valve position

40FC185 valve position

40FC186 valve position

40FC187 valve position

Top temperature

Kero flashpoint

Kero $90 \%$ point

Added diesel cloud point

Total gasoil flow

4 th side color

D-2 level

Splitter reboiler bypass flow

Stabilizer reboiler bypass flow

40FRC62 valve position

40FRC63 valve position

40FRC96 valve position

Total crude feed 

32. Furnace A duty
33. Furnace B duty
34. 40FZDTO:
Flash zone delta temperature
35. 40AR5/40TBPHSR:
HSR $90 \%$ point
36. 40PRC15:
Suction pressure $40 \mathrm{~K} 30$

At first a single variable step test approach was used for model identification. The step test took about 14 days with a control engineer on site around the clock. This high cost of step test is typical in current MPC projects. The identified model was used in the DMC controller and the control performance was not satisfactory. It was believed that model quality was one of the causes of control problems. This situation motivated the search for more effective and efficient identification approaches and the ASYM method was chosen for its promising features. The following are the results of ASYM identification.

\section{A. ASYM Identification Tests}

It was decided that 13 of the 19 MVs will be tested and their models identified using ASYM. PRBS signals are used as test signals. The average switch time of all the PRBS signals are set to 50 minutes. This means that the estimated time to steady state of the process is about two and half hours. The amplitudes of the PRBS signals are chosen such that they will generate data with good enough signal-to-noise ratio but will not disturb the product quality. Normally, most of the information needed for determining the amplitudes of PRBS signals can be obtained by interviewing experienced operators and operation engineers and by performing some pre-tests (short step tests). Two open-loop tests were designed and carried out:

Test 1: $\quad$ MV

40TRC50S
40FC184S
40FC185S
40FC186S
40FC187S
40FRC35S
40FRC79S
40FC170S
40FRC22S

Test 2: $\quad$ MV
PRBS average switch time

$50 \mathrm{~min}$.

$50 \mathrm{~min}$.

$50 \mathrm{~min}$.

$50 \mathrm{~min}$.

50 min.

Manual

$50 \mathrm{~min}$.

$50 \mathrm{~min}$.

$50 \mathrm{~min}$.

PRBS average switch time

$50 \mathrm{~min}$.

$50 \mathrm{~min}$.

$50 \mathrm{~min}$.

$50 \mathrm{~min}$.

Manual

Amplitude (top/top)
\[ \begin{array}{l}2{ }^{\circ} \mathrm{C} \\ 200 \mathrm{M}^{3} / \mathrm{D} \\ 200 \mathrm{M}^{3} / \mathrm{D} \\ 200 \mathrm{M}^{3} / \mathrm{D} \\ 200 \mathrm{M}^{3} / \mathrm{D} \\ 0.4 \mathrm{KM}^{3} / \mathrm{D} \\ 150 \mathrm{M}^{3} / \mathrm{D} \\ 150,100 \mathrm{M}^{3} / \mathrm{D} \\ 100 \mathrm{M}^{3} / \mathrm{D}\end{array} \]

Amplitude (top/top)
$0.2 \mathrm{KM}^{3} / \mathrm{D}$
$0.2 \mathrm{KM}^{3} / \mathrm{D}$
$0.3 \mathrm{KM}^{3} / \mathrm{D}$
$0.2 \mathrm{KM}^{3} / \mathrm{D}$
$0.6 \mathrm{KM}^{3} / \mathrm{D}$

Each test lasted for about two days, so the total test time is 4 days. There is also a control engineer on site around the clock during the tests. But this time his task is not to step the process manually, but rather to give advise to operators in case of too big disturbances. The tests did not cause any product quality problems. Figure 5.2 shows the plots of part of the 8 MVs during some of CVs during PRBS Test 1. During the test the operators have adjusted the average setpoints for many MVs in order to maintain stable unit operation. 
MVs of Test 1

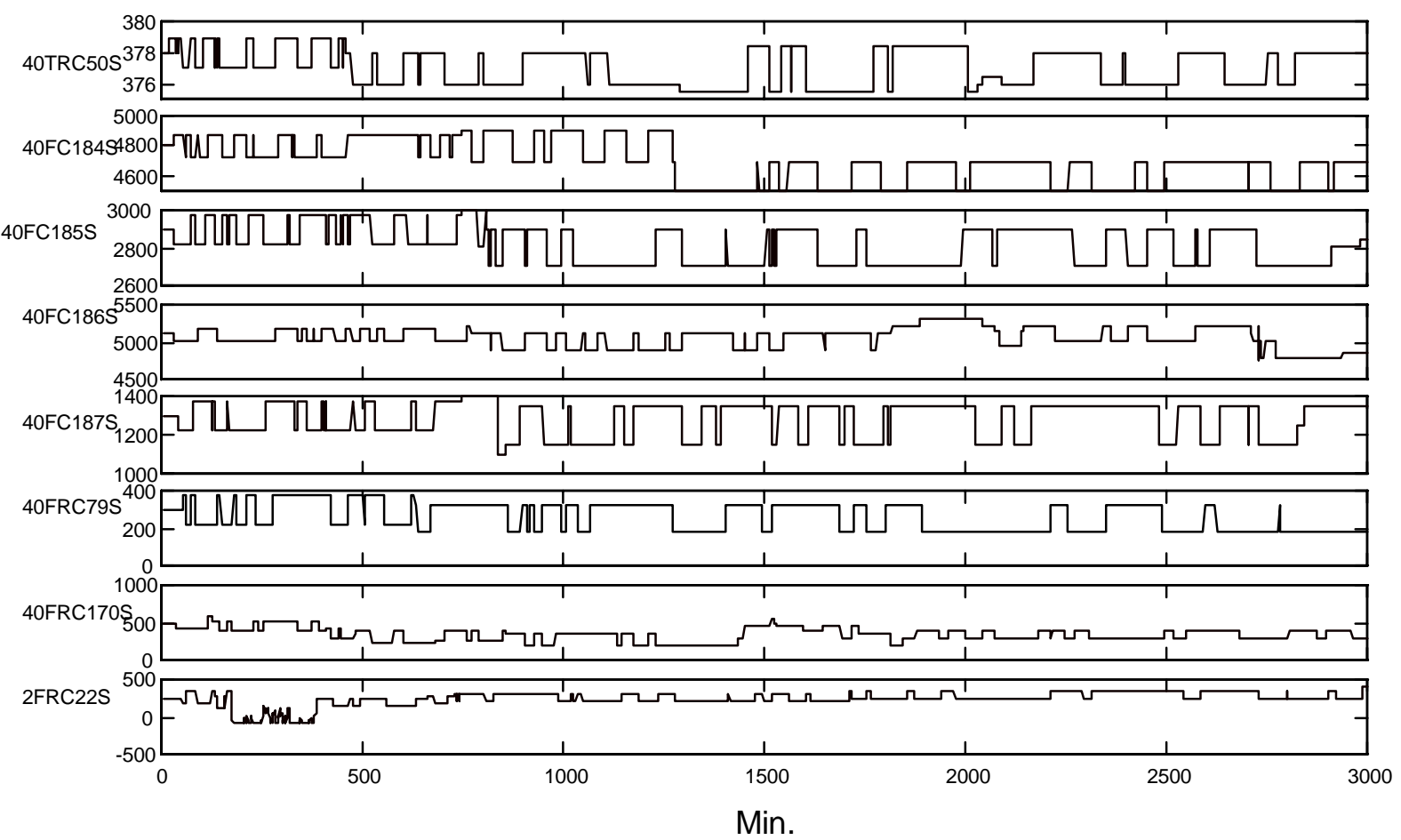

Part of the CVs of Test 1

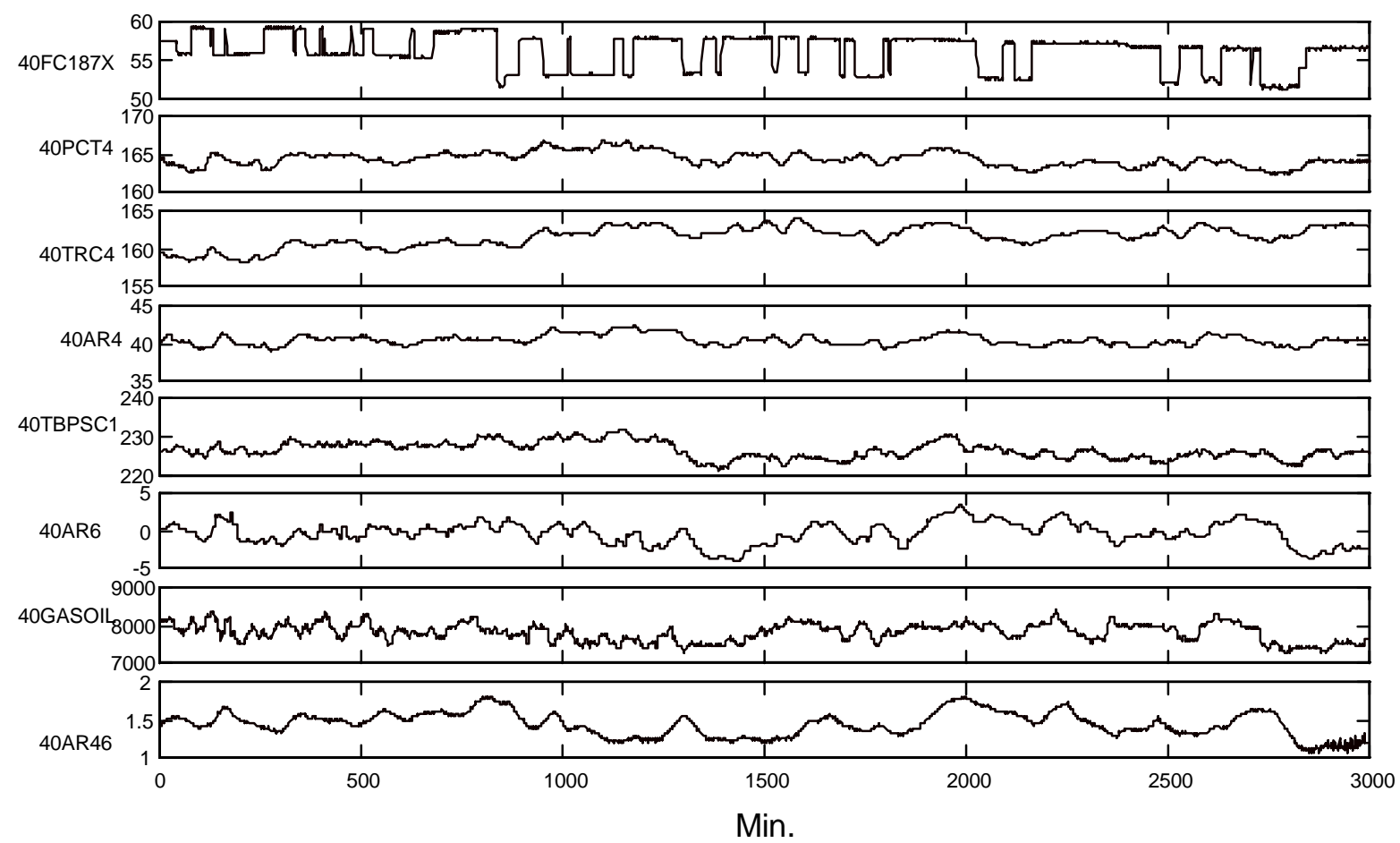

Figure 5.2 Trends of part of moved MVs and CVs during PRBS Test 1 


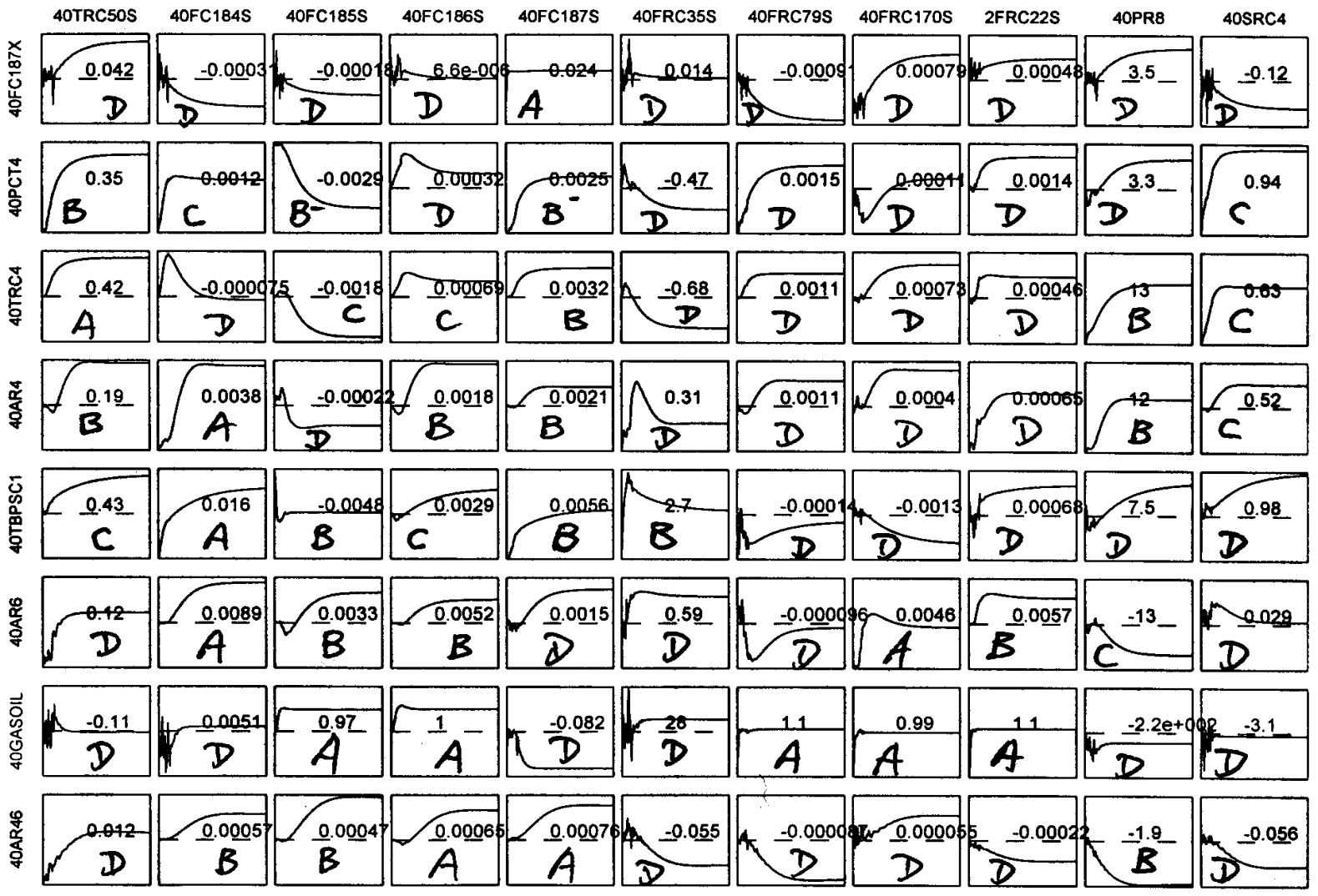

Time [min], total 200.

Figure 5.3 Step responses of the models of $8 \mathrm{CVs}$ from Test1

40TRC50S 40FC184S 40FC185S 40FC186S 40FC187S 40FRC35S 40FRC79S 40FRC170S 2FRC22S 40PR8

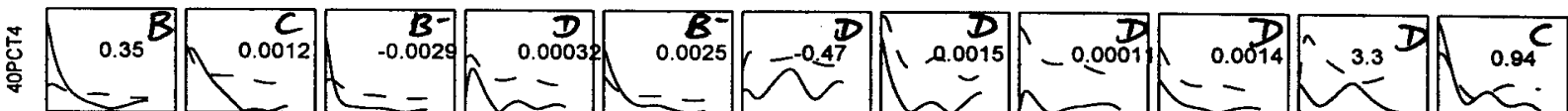

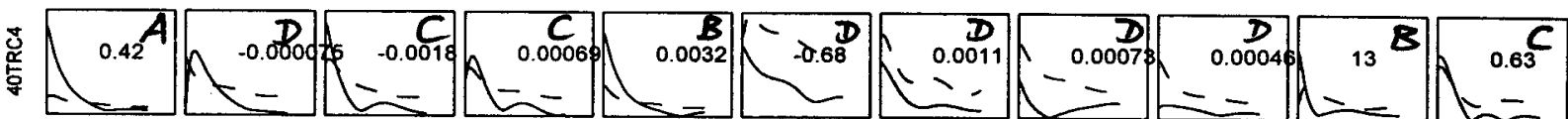

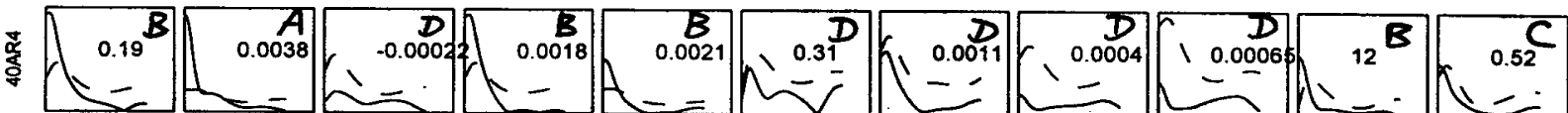

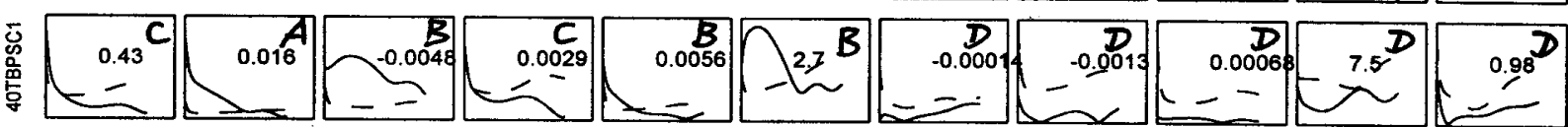

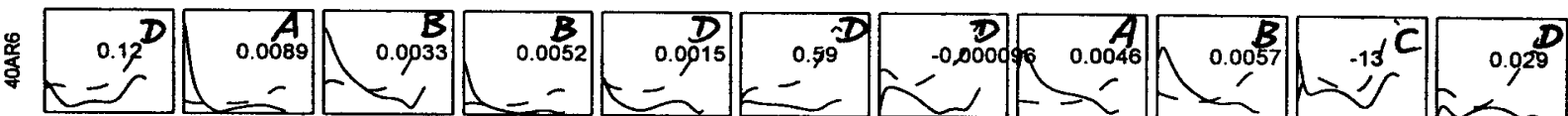

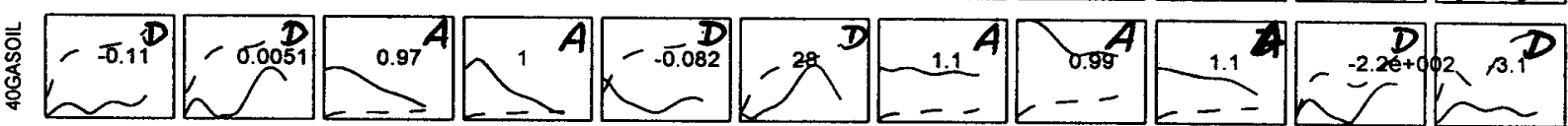

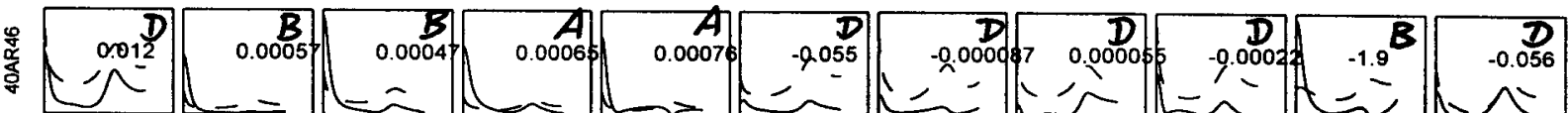

Norm. freq [ $\mathrm{rad} / \mathrm{min}]$, total 0.785398 .

Figure 5.4 Bode plots (solid) and error bounds (dashed). 


\section{B. Process Models and Model Validation}

In Figure 5.3 the model step responses of $8 \mathrm{CVs}$ for Test 1 are plotted, in Figure 5.4 their frequency responses and upper error bounds are plotted. The models are graded according to the relative size of their upper bounds. The result of model validation agreed very well with process knowledge. Model validation results using upper error bounds agreed very well with the process knowledge. Most of the A and B grade models are used in the DMC controller.

\section{DMC Commissioning and its Performance}

The ASYM models are very accurate for the working range tested. As a result the controller can be tuned very fast. This time, it took only two weeks to commission the atmospheric column DMC controller. The controller has been on-line for over a year. Control results are pointing at a much higher yield pattern at the expense of residue. The variance of the product qualities has been reduced dramatically.

\section{Conclusions and Perspectives}

MPC technology has been widely and successfully applied in the refinery and petrochemical industries. Current experience shows that process modelling and identification become a bottleneck during an MPC project, and most processes are identified using conventional single variable step test approach. In this work we have introduced the ASYM method to multivariable process identification for MPC and presented a simulation study and an industrial application. Successful results have been obtained during many other MPC projects using ASYM. When comparing to the conventional single variable step test approach the following improvements have been made by ASYM:

- The model quality is high due to maximum likelihood estimates and parametric models; and model validation is easy due to the use of upper error bounds.

- The disturbance to the product qualities is minimized due to the relative small signal amplitudes, fast movement and encouraged operator manual control action. Even better test conditions can be realised when closed-loop test is used.

- The cost of identification is significantly reduced. Reduction of the test time by over $60 \%$ has been realised. The MV movements are done by the computer and hence the control engineer is free from watching the tests continuously. Requires less users knowledge and experience on identification. This is because most of the steps of ASYM can be automated and trial-and-error is minimized.

A powerful and effective identification tool can make more process units justified for MPC applications.

When comparing to recently emerging methods of parameter estimation, ASYM provides systematic solutions to all the four identification problems for control, while other methods provide solutions to only part of the four problems. Systematic solutions of identification problems make automatic identification possible. We believe that automatic identification holds the key for wide spread applications of model based control such as MPC.

It is predicted that the new generation industrial MPC will use parametric models and observers (Qin and Badgwell, 1996). As we have seen, ASYM calculates parametric models for both process transfer function matrix and unmeasured disturbances. Future extensions of 
the ASYM method can be translating error bounds from frequency response to step response, identification for ill-conditioned processes, and adding non-linearity to the model set.

Acknowledgement. The author would like to thank Nerefco B.V., The Netherlands, for the permission of publication of the material about the crude unit DMC application. He also thanks the reviewers for their comments that helped to improve the presentation of the work.

\section{References}

Cott, B.J. (1995a). Introduction to the Process Identification Workshop at the 1992 Canadian Chemical Engineering Conference. J. Process Control, Vol. 5, No. 2, pp. 109-113.

Cott, B.J. (1995b). Summary of the Process Identification Workshop at the 1992 Canadian Chemical Engineering Conference. J. Process Control, Vol. 5, No. 2, pp 109-113.

Cutler, C.R. and R.B. Hawkins (1988). Application of a large predictive multivariable controller to a hydrocracher second stage reactor. Proceedings of American Control Conference, pp. 284-291.

Hjalmarsson, H., M. Gevers, F. de Bruyne (1996). For model-based control design, closedloop identification gives better performance. Automatica, Vol. 32, No. 12, pp. 1659-1673.

Kouvaritakis, B. and H. Latchman (1985). Necessary and sufficient stability criterion for systems with structured uncertainties: the major principal direction alignment principle. Int. J. Control, Vol. 42, No. 3, pp. 575-598.

Ljung, L. (1985). Asymptotic variance expressions for identified black-box transfer function models. IEEE Trans. Autom. Control, Vol. AC-30, pp. 834-844.

Ljung. L. (1987). System Identification: Theory for the User. Prentice-Hall, Englewood Cliffs, N.J.

Ljung, L. and Z.D. Yuan (1985). Asymptotic properties of black-box identification of transfer functions. IEEE Trans. Autom. Control, Vol. AC-30, pp.514-530.

Qin, S.J. and T.A. Badgwell (1996). An overview of industrial model predictive control technology, Internet: www.che.utexas.edu/ qin/cpcv/cpcv14.html.

Richalet, J. (1993). Industrial applications of model based predictive control. Automatica, Vol. 29, No. 5, pp. 1251-1274.

Treiber, S, M. De Tremblay, M. Bell, B. Lines and D. Hartlen (1992). Modelling and control design of a polyethylene reactor. Proceedings of American Control Conference.

Wahlberg, B. (1989). Model reduction of high-order estimated models: the asymptotic ML approach. Int. J. Control, Vol. 49, No. 1, pp. 169-192.

Zhu, Y.C. (1989). Black-box identification of MIMO transfer functions: asymptotic properties of prediction error models. Int. J. Adaptive Control and Signal Processing, Vol. 3, pp. 357-373.

Zhu, Y.C., A.C.P.M. Backx and P. Eykhoff (1991). Multivariable process identification based on frequency domain measures. Proceedings of 30th IEEE Conference on Decision and Control, Brighton, December, 1991, pp. 303-308.

Zhu, Y.C. and T. Backx (1993). Identification of Multivariable Industrial Processes: for Simulation, Diagnosis and Control. Springer-Verlag, London.

Zhu, Y.C. (1994). A frequency domain criterion for MIMO model structure selection. Preprints of 10th IFAC Symposium on System Identification, 4-6 July 1994, Copenhagen, Denmark, vol. 2, pp. 516-521. 
Zhu Y.C., M. van Wijck, E. Janssen, A.J.M. Graaf, C.H. van Aalst and L. Kieviet (1997). "Crude unit identification for MPC using ASYM method", Proceedings of American Control Conference, pp. 3395-3399.

Zhu, Y.C. and X.H. Ge (1997). Tai-Ji ID: automatic system identification package for model based process control. Journal A, Vol. 38, No. 3, pp 42-45. 